Article

\title{
On a Periodic Boundary Value Problem for a Fractional-Order Semilinear Functional Differential Inclusions in a Banach Space
}

\author{
Mikhail Kamenski ${ }^{1}$, Valeri Obukhovskii ${ }^{2}$, Garik Petrosyan ${ }^{3}$ and Jen-Chih Yao ${ }^{4, *}$ \\ 1 Faculty of Mathematics, Voronezh State University, Voronezh 394018, Russia; mikhailkamenski@mail.ru \\ 2 Faculty of Physics and Mathematics, Voronezh State Pedagogical University, Voronezh 394043, Russia; \\ valerio-ob2000@mail.ru \\ 3 Research Center of Voronezh State University of Engineering Technologies and Faculty of Physics and \\ Mathematics, Voronezh State Pedagogical University, Voronezh 394043, Russia; garikpetrosyan@yandex.ru \\ 4 Research Center for Interneural Computing, China Medical University, Taichung 40447, Taiwan \\ * Correspondence: yaojc@mail.cmu.edu.tw
}

Received: 28 October 2019; Accepted: 18 November 2019; Published: 23 November 2019

\begin{abstract}
We consider the periodic boundary value problem (PBVP) for a semilinear fractional-order delayed functional differential inclusion in a Banach space. We introduce and study a multivalued integral operator whose fixed points coincide with mild solutions of our problem. On that base, we prove the main existence result (Theorem 4). We present an example dealing with existence of a trajectory for a time-fractional diffusion type feedback control system with a delay satisfying periodic boundary value condition.
\end{abstract}

Keywords: fractional functional differential inclusion; semilinear functional differential inclusion; periodic boundary value problem; time-fractional diffusion type feedback control system; fixed point; condensing map; measure of noncompactness

MSC: Primary: 34G25; Secondary: 34K09; 34K13; 34K37; 35B10; 47H04; 47H08; 47H10

\section{Introduction}

At the present time, the theory of differential equations and inclusions of fractional order is the subject of an active study for a large number of researchers. One of the main reasons for this interest is caused by important applications of this theory in physics, engineering, biology, economics, and other sciences (see, e.g., [1-4] and the references therein). It should be mentioned in this connection that fractional order models provide an effective and convenient machinery for the description of systems with memory and hereditary properties.

In the present paper, for a semilinear fractional-order functional differential inclusion in a separable Banach space $E$ of the form

$$
{ }^{C} D^{q} x(t) \in A x(t)+F\left(t, x_{t}\right), t \in[0, T],
$$

we consider the problem of existence of a mild solution to this inclusion satisfying the periodic boundary value condition (PBVP). Here, ${ }^{C} D^{q}$ denotes the Caputo fractional derivative of the order $0<q<1, A: D(A) \subset E \rightarrow E$ is the infinitesimal generator of a bounded $C_{0}$-semigroup, $F:[0, T] \times$ $C([-h, 0] ; E) \multimap E$ is a multivalued nonlinearity and the function $x_{t}$ describes the prehistory of the solution at the moment $t \in[0, T]$, i.e., $x_{t}(s)=x(t+s), s \in[-h, 0], 0<h<T$. It is worth noting that the introducing into consideration of the delay of the trajectory allows, additionally to the application 
of the fractional derivative, to reflect the dependence of the current state of a system on its previous characteristics.

Among a large amount of papers dedicated to fractional-order equations and inclusions in Banach spaces, let us mention works [5-15] where existence results of various types were obtained. In particular, in the authors' paper [6], the periodic boundary value problem for fractional-order semilinear differential inclusions in Banach spaces was studied by the method of translation multioperator along the trajectories of the inclusion. However, this method can not be extended directly to the case of functional differential inclusions. For this reason, in the present paper, we apply for the solving of the PBVP the method of integral multioperators, combined with the theory of condensing multimaps and the theory of Mittag-Leffler functions.

The paper is organized in the following way. In the next section, we present necessary notions and facts from the fractional and multivalued analysis as well as from the theory of condensing maps. In Section 3, we study the PBVP for a semilinear fractional-order functional differential inclusion with delay in a Banach space. We introduce and study a multivalued integral operator whose fixed points coincide with mild solutions of our problem. On that base, we prove the main existence result (Theorem 4). In the last section, we consider an example dealing with existence of a trajectory for a time-fractional diffusion type feedback control system with delay satisfying the periodic boundary .

\section{Preliminaries}

\subsection{Differential Equations of Fractional Order}

Recall some notions and definitions which we will need in the sequel (details can be found, e.g., in $[1,3,4])$.

Let $E$ be a real Banach space.

Definition 1. The Riemann-Liouville fractional derivative of the order $q \in(0,1)$ of a continuous function $g:[0, a] \rightarrow E$ is the function $D^{q} g$ of the following form:

$$
D^{q} g(t)=\frac{1}{\Gamma(1-q)} \frac{d}{d t} \int_{0}^{t}(t-s)^{-q} g(s) d s
$$

provided the right-hand side of this equality is well defined.

Here, $\Gamma$ is the Euler gamma-function

$$
\Gamma(r)=\int_{0}^{\infty} s^{r-1} e^{-s} d s
$$

Definition 2. The Caputo fractional derivative of the order $q \in(0,1)$ of a continuous function $g:[0, a] \rightarrow E$ is the function ${ }^{C} D^{q} g$ defined in the following way:

$$
{ }^{C} D^{q} g(t)=\left(D^{q}(g(\cdot)-g(0))\right)(t)
$$

provided the right-hand side of this equality is well defined.

Definition 3. A function of the form

$$
E_{q, \beta}(z)=\sum_{n=0}^{\infty} \frac{z^{n}}{\Gamma(q n+\beta)}, \quad q, \beta>0, z \in \mathbb{C}
$$

is called the Mittag-Leffler function. 
Denote $E_{q, 1}$ by $E_{q}$. Notice that from the relations (see, e.g., [16]):

$$
E_{q}(-z)=\int_{0}^{\infty} \xi_{q}(\theta) e^{-z \theta} d \theta
$$

and

$$
E_{q, q}(-z)=\int_{0}^{\infty} q \theta \xi_{q}(\theta) e^{-z \theta} d \theta
$$

where

$$
\begin{gathered}
\xi_{q}(\theta)=\frac{1}{q} \theta^{-1-\frac{1}{q}} \Psi_{q}\left(\theta^{-1 / q}\right), \\
\Psi_{q}(\theta)=\frac{1}{\pi} \sum_{n=1}^{\infty}(-1)^{n-1} \theta^{-q n-1} \frac{\Gamma(n q+1)}{n !} \sin (n \pi q), \theta \in \mathbb{R}_{+} .
\end{gathered}
$$

It follows that

$$
E_{q}(\tau)>0, E_{q, q}(\tau)>0 \text { for } \tau<0 .
$$

$\operatorname{Remark} 1$ (See, e.g., $[4,14]) . \xi_{q}(\theta) \geq 0, \int_{0}^{\infty} \xi_{q}(\theta) d \theta=1, \int_{0}^{\infty} \theta \xi_{q}(\theta) d \theta=\frac{1}{\Gamma(q+1)}$

In the sequel, we will need the following relations (see [17])

$$
\begin{gathered}
E_{q, \beta}(z)=\frac{1}{\Gamma(\beta)}+z E_{q, \beta+q}(z), \\
\int_{0}^{z} t^{\beta-1} E_{q, \beta}\left(\lambda t^{q}\right) d t=z^{\beta} E_{q, \beta+1}\left(\lambda z^{q}\right) .
\end{gathered}
$$

2.2. Measures of Noncompactness and Condensing Maps

Let $\mathcal{E}$ be a Banach space. Introduce the following notation:

- $P(\mathcal{E})=\{A \subseteq \mathcal{E}: A \neq \varnothing\}$;

- $\quad \operatorname{Pb}(\mathcal{E})=\{A \in P(\mathcal{E}): A$ is bounded $\}$;

- $\operatorname{Pv}(\mathcal{E})=\{A \in P(\mathcal{E}): A$ is convex $\}$;

- $K(\mathcal{E})=\{A \in P b(\mathcal{E}): A$ is compact $\}$;

- $K v(\mathcal{E})=\operatorname{Pv}(\mathcal{E}) \cap K(\mathcal{E})$.

Definition 4 (See, e.g., [18]). Let $(\mathcal{A}, \geq)$ be a partially ordered set. A function $\beta: \mathrm{Pb}(\mathcal{E}) \rightarrow \mathcal{A}$ is called the measure of noncompactness $(M N C)$ in $\mathcal{E}$ if for each $\Omega \in \mathrm{Pb}(\mathcal{E})$ we have:

$$
\beta(\overline{\mathrm{CO}} \Omega)=\beta(\Omega),
$$

where $\overline{\mathrm{co}} \Omega$ denotes the closure of the convex hull of $\Omega$.

A measure of noncompactness $\beta$ is called:

(1) monotone if for each $\Omega_{0}, \Omega_{1} \in \operatorname{Pb}(\mathcal{E}), \Omega_{0} \subseteq \Omega_{1}$ implies $\beta\left(\Omega_{0}\right) \leq \beta\left(\Omega_{1}\right)$;

(2) nonsingular if for each $a \in \mathcal{E}$ and each $\Omega \in \operatorname{Pb}(\mathcal{E})$ we have $\beta(\{a\} \cup \Omega)=\beta(\Omega)$.

If $\mathcal{A}$ is a cone in a Banach space, the MNC $\beta$ is called:

(3) regular if $\beta(\Omega)=0$ is equivalent to the relative compactness of $\Omega \in \operatorname{Pb}(\mathcal{E})$;

(4) real if $\mathcal{A}$ is the set of all real numbers $\mathbb{R}$ with the natural ordering;

(5) algebraically semiadditive if $\beta\left(\Omega_{0}+\Omega_{1}\right) \leq \beta\left(\Omega_{0}\right)+\beta\left(\Omega_{1}\right)$ for every $\Omega_{0}, \Omega_{1} \in \operatorname{Pb}(\mathcal{E})$. 
As the example of a real MNC obeying all above properties, we can consider the Hausdorff $\operatorname{MNC} \chi(\Omega)$ :

$$
\chi(\Omega)=\inf \{\varepsilon>0, \text { for which } \Omega \text { has a finite } \varepsilon \text {-net in } \mathcal{E}\} .
$$

Notice that the Hausdorff MNC satisfies the semi-homogeneity condition, i.e.,:

$$
\chi(\lambda \Omega)=|\lambda| \chi(\Omega),
$$

for every $\lambda \in \mathbb{R}$ and $\Omega \in \mathrm{P}(\mathcal{E})$. More generally, if $\mathcal{L}: \mathcal{E} \rightarrow \mathcal{E}$ is a bounded linear operator, then

$$
\chi(\mathcal{L}(\Omega))=\|\mathcal{L}\| \chi(\Omega)
$$

for every $\Omega \in \mathrm{P}(\mathcal{E})$.

Recall that the norm of a set $M \in \mathrm{Pb}(\mathcal{E})$ is defined by the formula:

$$
\|M\|=\sup _{x \in M}\|x\|_{\mathcal{E}}
$$

Definition 5 (See, e.g., [18]). Let $X$ be a closed subset of $\mathcal{E} ; \beta$ a MNC in $\mathcal{E}$. A multivalued map (multimap) $\mathcal{F}: X \rightarrow K(\mathcal{E})$ is called condensing w.r.t. $\beta$ (or $\beta$-condensing) if for every $\Omega \in P b(X)$ which is not relatively compact we have:

$$
\beta(F(\Omega)) \nsupseteq \beta(\Omega) .
$$

Definition 6 (See, e.g., [18-20]). Let $X$ be a metric space. A multimap $\mathcal{F}: X \rightarrow P(\mathcal{E})$ is called upper semicontinuous (u.s.c.) if

$$
\mathcal{F}^{-1}(V)=\{x \in X: \mathcal{F}(x) \subset V\}
$$

is an open subset of $X$ for each open set $V \subset \mathcal{E}$.

Theorem 1 (Cf. Corollary 3.3.1 [18]). Let $\mathcal{M}$ be a convex closed subset of $\mathcal{E}$ and $\mathcal{F}: \mathcal{M} \rightarrow \operatorname{Kv}(\mathcal{M})$ a u.s.c. $\beta$-condensing multimap, where $\beta$ is a nonsingular MNC in $\mathcal{E}$. Then, the fixed point set Fix $\mathcal{F}=\{x: x \in \mathcal{F}(x)\}$ is non-empty.

Recall some notions (see, e.g., $[18,20])$. Let $E$ be a Banach space.

Definition 7. For a given $1 \leq p \leq \infty$, a multifunction $G:[0, \tau] \rightarrow K(E)$ is called:

- $\quad L^{p}$-integrable if it admits an $L^{p}$-Bochner integrable selection, i.e., there exists a function $g \in L^{p}((0, \tau) ; E)$ such that $g(t) \in G(t)$ for a.e. $t \in[0, \tau]$;

- $\quad L^{p}$-integrably bounded if there exists a function $\xi \in L^{p}((0, \tau))$ such that

$$
\|G(t)\| \leq \xi(t)
$$

for a.e. $t \in[0, \tau]$.

The set of all $L^{p}$-integrable selections of a multifunction $G:[0, \tau] \rightarrow K(E)$ is denoted by $\mathcal{S}_{G}^{p}[0, \tau]$.

Definition 8. The integral of an $L^{p}$-integrable multifunction $G:[0, \tau] \rightarrow K(E)$ is defined in the following way:

$$
\int_{0}^{\tau} G(s) d s=\left\{\int_{0}^{\tau} f(s) d s: f \in \mathcal{S}_{G}^{p}[0, \tau]\right\} .
$$

In the sequel, we will need the following important property on the $\chi$-estimation of the integral of a multifunction. 
Lemma 1 (See Theorem 4.2.3 in [18]). Let E be a separable Banach space and $G:[0, \tau] \rightarrow K(E)$ an integrable, integrably bounded multifunction such that

$$
\chi(G(t) \leq v(t) \text { for a.e. } t \in[0, \tau]
$$

where $\chi$ is the Hausdorff MNC in $E$ and $v(\cdot) \in L_{+}^{1}(0, \tau)$. Then,

$$
\chi\left(\int_{0}^{\tau} G(s) d s\right) \leq \int_{0}^{\tau} v(s) d s .
$$

\section{Existence Result}

For a semilinear fractional-order differential inclusion in a separable Banach space $E$ of the form

$$
{ }^{C} D^{q} x(t) \in A x(t)+F\left(t, x_{t}\right), t \in[0, T],
$$

we consider the problem (PBVP) of existence of a mild solution satisfying the following periodic boundary value condition

$$
x_{0}=x_{T}
$$

under the next basic assumptions.

As earlier, the symbol ${ }^{C} D^{q} x$ denotes the Caputo fractional derivative of order $q \in(0,1)$. We suppose that the linear operator $A$ satisfies condition

(A) $A: D(A) \subseteq E \rightarrow E$ is a linear closed (not necessarily bounded) operator generating a bounded $C_{0}$-semigroup $\{U(t)\}_{t \geq 0}$ of linear operators in $E$.

In the sequel, we will use the notation $\mathcal{C}:=C([-h, 0] ; E)$.

We will assume that the multivalued nonlinearity $F:[0, T] \times \mathcal{C} \rightarrow K v(E)$ obeys the following conditions:

$(F 1)$ for each $\xi \in \mathcal{C}$ the multifunction $F(\cdot, \xi):[0, T] \rightarrow K v(E)$ admits a measurable selection;

(F2) for a.e. $t \in[0, T]$ the multimap $F(t, \cdot): E \rightarrow K v(E)$ is u.s.c.;

(F3) there exist functions $\alpha, \gamma \in L_{+}^{\infty}([0, T])$ such that, for each $\xi \in \mathcal{C}$, we have

$$
\|F(t, \xi)\|_{E} \leq \alpha(t)+\gamma(t)\|\xi\|_{\mathcal{C}} \text { for a.e. } t \in[0, T],
$$

(F4) there exists a function $\mu \in L^{\infty}([0, T])$ such that for each bounded set $\Delta \subset \mathcal{C}$ we have:

$$
\chi(F(t, \Delta)) \leq \mu(t) \varphi(\Delta),
$$

for a.e. $t \in[0, T]$, where $\varphi(\Delta)=\sup _{s \in[-h, 0]} \chi(\Delta(s)), \chi$ is the Hausdorff MNC in $E, \Delta(s)=$ $\{y(s): y \in \Delta\}$.

For a given $x \in C([-h, T] ; E)$ consider the multifunction

$$
\Phi:[0, T] \rightarrow K v(E), \quad \Phi(t)=F\left(t, x_{t}\right) .
$$

From above conditions (F1)-(F3), it follows (see, e.g., [18] Theorem 1.3.5) that the multifunction $\Phi$ is $L^{\infty}$-integrable and, therefore, the superposition multioperator $\mathcal{P}_{F}^{\infty}: C([-h, T] ; E) \rightarrow P\left(L^{\infty}([0, T] ; E)\right)$ can be defined in the following way:

$$
\mathcal{P}_{F}^{\infty}(x)=\mathcal{S}_{\Phi}^{\infty}
$$


Now, we consider the operator functions defined in the following way:

$$
\begin{gathered}
\mathcal{G}(t)=\int_{0}^{\infty} \xi_{q}(\theta) U\left(t^{q} \theta\right) d \theta, \\
\mathcal{T}(t)=q \int_{0}^{\infty} \theta \xi_{q}(\theta) U\left(t^{q} \theta\right) d \theta,
\end{gathered}
$$

where the function $\xi_{q}(\theta)$ is given by (2) and (3).

Remark 2. In a scalar case $E=\mathbb{R}$ and $U(t)=e^{-\eta t}$ with $\eta>0$ :

$$
\mathcal{G}(t)=E_{q}\left(-\eta t^{q}\right), \quad \mathcal{T}(t)=E_{q, q}\left(-\eta t^{q}\right), \quad t \in[0, T] .
$$

Lemma 2 (See [4,14]). The operator functions $\mathcal{G}$ and $\mathcal{T}$ possess the following properties:

(1) for each $t \in[0, T], \mathcal{G}(t)$ and $\mathcal{T}(t)$ are linear bounded operators and moreover, if the semigroup $U(t)$ satisfies the estimate

$$
\|U(t)\| \leq e^{-\eta t}, \quad t \geq 0
$$

with $\eta>0$, then

$$
\begin{gathered}
\|\mathcal{G}(t)\| \leq E_{q}\left(-\eta t^{q}\right) \leq 1, \quad t \in[0, T], \\
\|\mathcal{T}(t)\| \leq E_{q, q}\left(-\eta t^{q}\right) \leq \frac{q}{\Gamma(1+q)}, \quad t \in[0, T] ;
\end{gathered}
$$

(2) the operator functions $\mathcal{G}(\cdot)$ and $\mathcal{T}(\cdot)$ are strongly continuous, i.e., functions $t \in[0, T] \rightarrow \mathcal{G}(t) x$ and $t \in[0, T] \rightarrow \mathcal{T}(t) x$ are continuous for each $x \in E$.

Definition 9. A mild solution of inclusion (7) is a function $x \in C([-h, T] ; E)$ satisfying

$$
x(t)=\mathcal{G}(t) x(0)+\int_{0}^{t}(t-s)^{q-1} \mathcal{T}(t-s) \phi(s) d s, \quad t \in[0, T],
$$

where $\phi \in \mathcal{P}_{F}^{\infty}(x)$,

In what follows, we will assume that the next condition holds true:

$$
1 \notin s p[\mathcal{G}(T)]
$$

Consider the multioperator $G: C([-h, T] ; E) \rightarrow P(C([-h, T] ; E))$, given in the following way:

$$
G(x)=\{y\}
$$

for all functions $y$ defined as

$$
\left\{\begin{array}{lr}
y(t)=\mathcal{G}(t)(I-\mathcal{G}(T))^{-1} \int_{0}^{T}(T-s)^{q-1} \mathcal{T}(T-s) \phi(s) d s+ \\
\int_{0}^{t}(t-s)^{q-1} \mathcal{T}(t-s) \phi(s) d s, & t \in[0, T], \\
y(s)=y(T-s), & s \in[-h, 0]
\end{array}\right.
$$

where $\phi \in \mathcal{P}_{F}^{\infty}(x)$.

The well posedness of the operator $G$ follows from the next assertion.

Lemma 3. If $y \in G(x)$ for some $x \in C([-h, T] ; E)$, then $y(0)=y(T)$ and hence $y_{0}=y_{T}$. 
Proof. In fact,

$$
\begin{gathered}
y(T)=\mathcal{G}(T)(I-\mathcal{G}(T))^{-1} \int_{0}^{T}(T-s)^{q-1} \mathcal{T}(T-s) \phi(s) d s+\int_{0}^{T}(T-s)^{q-1} \mathcal{T}(T-s) \phi(s) d s= \\
=\left(\mathcal{G}(T)(I-\mathcal{G}(T))^{-1}+I\right) \int_{0}^{T}(T-s)^{q-1} \mathcal{T}(T-s) \phi(s) d s= \\
=(I-\mathcal{G}(T))^{-1} \int_{0}^{T}(T-s)^{q-1} \mathcal{T}(T-s) \phi(s) d s=y(0) .
\end{gathered}
$$

Equality $y_{0}=y_{T}$ then follows from the definition of $G$.

Theorem 2. Fixed points of the multioperator $G$ coinside with mild solutions to problems (7) and (8).

\section{Proof.}

(i) If $x$ is a solution of problems (7) and (8), then, for $t \in[0, T]$, it has the form

$$
x(t)=\mathcal{G}(t) x(0)+\int_{0}^{t}(t-s)^{q-1} \mathcal{T}(t-s) \phi(s) d s,
$$

where $\phi \in \mathcal{P}_{F}^{\infty}(x)$. From condition (8), it follows that

$$
x(0)=x(T)=\mathcal{G}(T) x(0)+\int_{0}^{T}(T-s)^{q-1} \mathcal{T}(T-s) \phi(s) d s,
$$

implying

$$
x(0)=(I-\mathcal{G}(T))^{-1} \int_{0}^{T}(T-s)^{q-1} \mathcal{T}(T-s) \phi(s) d s,
$$

that yields, for $t \in[0, T]$,

$$
x(t)=\mathcal{G}(t)(I-\mathcal{G}(T))^{-1} \int_{0}^{T}(T-s)^{q-1} \mathcal{T}(T-s) \phi(s) d s+\int_{0}^{t}(t-s)^{q-1} \mathcal{T}(t-s) \phi(s) d s,
$$

that is $x \in$ FixG.

(ii) Conversely, let $x \in$ FixG; then, it satisfies, for $t \in[0, T]$, Equation (15) with $\phi \in \mathcal{P}_{F}^{\infty}(x)$, whence it is a mild solution of inclusion (7). The validity of condition (8) follows from Lemma 3.

Let us consider some topological properties of the multioperator $G$.

Lemma 4. The multioperator $G$ is u.s.c. and has compact values.

Proof. It is clear that it is sufficient to prove the assertion for the multioperator $G$ whose values are naturally restricted to the space $C([0, T] ; E)$. Let us denote this restriction as $\widetilde{G}$.

The multioperator $\widetilde{G}: C(-h, T] ; E) \rightarrow P(C([0, T] ; E))$ may be represented in the form of the following composition:

$$
\widetilde{G}(x)=\sigma \circ \bar{e} \circ \bar{g} \circ S \circ \mathcal{P}_{F}^{\infty}(x),
$$

where

$$
\begin{gathered}
S: L^{\infty}([0, T] ; E) \rightarrow C([0, T] ; E) \\
S(\phi)(t)=\int_{0}^{t}(t-s)^{q-1} \mathcal{T}(t-s) \phi(s) d s ; \\
\bar{g}: C([0, T] ; E) \rightarrow C([0, T] ; E) \times C([0, T] ; E), \\
\bar{g}(u)=(u, u) ;
\end{gathered}
$$




$$
\begin{gathered}
\bar{e}: C([0, T] ; E) \times C([0, T] ; E) \rightarrow C([0, T] ; E) \times C([0, T] ; E), \\
\bar{e}(u, v)=(w, v), \\
w(t)=\mathcal{G}(t)(I-\mathcal{G}(T))^{-1} u(T) ;
\end{gathered}
$$

and

$$
\begin{gathered}
\sigma: C([0, T] ; E) \times C([0, T] ; E) \rightarrow C([0, T] ; E), \\
\sigma(u, v)=u+v .
\end{gathered}
$$

From the results of the work [5], it is known that the multioperator $S \circ \mathcal{P}_{F}^{\infty}$ is u.s.c. with compact values and now the assertion follows from the fact that each of $\bar{g}, \bar{e}$, and $\sigma$ is a bounded linear operator.

To prove that the multioperator $G$ is condensing, consider the cone

$$
\mathbb{R}_{+}^{2}=\left\{\zeta=\left(\zeta_{1}, \zeta_{2}\right): \zeta_{1} \geq 0, \zeta_{2} \geq 0\right\}
$$

endowed with the natural ordering and introduce in the space $C([-h, T] ; E)$ the vector measure of noncompactness

$$
v: P(C([-h, T] ; E)) \rightarrow \mathbb{R}_{+}^{2}
$$

defined as

$$
v(\Omega)=\left(\varphi(\Omega), \bmod _{C}(\Omega)\right),
$$

where $\varphi(\Omega)$ is the module of fiber noncompactness

$$
\varphi(\Omega)=\sup _{t \in[-h, T]} \chi(\{y(t): y \in \Omega\})
$$

and the second component is the module of equicontinuity:

$$
\bmod _{C}(\Omega)=\lim _{\delta \rightarrow 0} \sup _{u \in \Omega} \max _{\left|t_{1}-t_{2}\right| \leq \delta}\left\|u\left(t_{1}\right)-u\left(t_{2}\right)\right\| .
$$

Theorem 3. Under assumptions ( $A)$, (F1)-(F4), suppose, additionally, that

(A1) the semigroup U satisfies estimate (9) for some $\eta>0$.

If

$$
\frac{\|\mu\|_{\infty}}{\eta}<1
$$

where $\mu(\cdot)$ is the function from condition (F4), then the multioperator $G$ is v-condensing.

Proof. Let $\Omega \subset C([-h, T] ; E)$ be a nonempty bounded set such that

$$
v(G(\Omega)) \geq v(\Omega) .
$$

Let us show that $\Omega$ is relatively compact.

From (19), it follows that

$$
\varphi(G(\Omega)) \geq \varphi(\Omega) .
$$

Let $0 \leq t \leq T$. By using estimates (10) and (11) and property (F4), and denoting, for $0 \leq s \leq T$, $\Omega_{s} \subset \mathcal{C}$,

$$
\Omega_{s}=\left\{x_{s}: x \in \Omega\right\},
$$

we get

$$
\chi(G(\Omega)(t)) \leq
$$




$$
\begin{gathered}
\leq \chi\left(\mathcal{G}(t)(I-\mathcal{G}(T))^{-1} \int_{0}^{T}(T-s)^{q-1} \mathcal{T}(T-s) F\left(s, \Omega_{s}\right) d s+\int_{0}^{t}(t-s)^{q-1} \mathcal{T}(t-s) F\left(s, \Omega_{s}\right) d s\right) \leq \\
\leq \frac{E_{q}\left(-\eta t^{q}\right)}{1-E_{q}\left(-\eta T^{q}\right)} \int_{0}^{T}(T-s)^{q-1} E_{q, q}\left(-\eta(T-s)^{q}\right) \mu(s) \varphi\left(\Omega_{s}\right) d s+ \\
\quad+\int_{0}^{t}(t-s)^{q-1} E_{q, q}\left(-\eta(t-s)^{q}\right) \mu(s) \varphi\left(\Omega_{s}\right) d s \leq \\
\leq \frac{E_{q}\left(-\eta t^{q}\right)}{1-E_{q}\left(-\eta T^{q}\right)}\|\mu\|_{\infty} \sup _{t \in[-h, T]} \chi(\Omega(t)) \int_{0}^{T}(T-s)^{q-1} E_{q, q}\left(-\eta(T-s)^{q}\right) d s+ \\
\quad+\|\mu\|_{\infty} \sup _{t \in[-h, T]} \chi(\Omega(t)) \int_{0}^{t}(t-s)^{q-1} E_{q, q}\left(-\eta(t-s)^{q}\right) d s .
\end{gathered}
$$

For further estimation of $\chi(G(\Omega)(t)), t \in[0, T]$, let us evaluate the integrals in the last expression by means of Formula (6):

$$
\begin{gathered}
\int_{0}^{T}(T-s)^{q-1} E_{q, q}\left(-\eta(T-s)^{q}\right) d s=-\int_{0}^{T}(T-s)^{q-1} E_{q, q}\left(-\eta(T-s)^{q}\right) d(T-s)= \\
=\int_{0}^{T} y^{q-1} E_{q, q}\left(-\eta y^{q}\right) d y=T^{q} E_{q, q+1}\left(-\eta T^{q}\right) .
\end{gathered}
$$

Similarly, we obtain

$$
\int_{0}^{t}(t-s)^{q-1} E_{q, q}\left(-\eta(t-s)^{q}\right) d s=t^{q} E_{q, q+1}\left(-\eta t^{q}\right) .
$$

Now, notice that, if we will take $\beta=1$ in Formula (5), we have

$$
E_{q}\left(-\eta t^{q}\right)=\frac{1}{\Gamma(1)}-\eta t^{q} E_{q, q+1}\left(-\eta t^{q}\right)=1-\eta t^{q} E_{q, q+1}\left(-\eta t^{q}\right) .
$$

Thus, we get the following equalities

$$
\begin{gathered}
\int_{0}^{T}(T-s)^{q-1} E_{q, q}\left(-\eta(T-s)^{q}\right) d s=T^{q} \frac{1}{\eta T^{q}}\left(1-E_{q}\left(-\eta T^{q}\right)\right)=\frac{1}{\eta}\left(1-E_{q}\left(-\eta T^{q}\right)\right), \\
\int_{0}^{t}(t-s)^{q-1} E_{q, q}\left(-\eta(t-s)^{q}\right) d s=\frac{1}{\eta}\left(1-E_{q}\left(-\eta t^{q}\right)\right) .
\end{gathered}
$$

Thus, for $t \in[0, T]$, we obtain

$$
\begin{gathered}
\chi(G(\Omega)(t)) \leq \frac{E_{q}\left(-\eta t^{q}\right)}{1-E_{q}\left(-\eta T^{q}\right)}\|\mu\|_{\infty} \sup _{t \in[-h, T]} \chi(\Omega(t)) \frac{1}{\eta}\left(1-E_{q}\left(-\eta T^{q}\right)\right)+ \\
\quad+\|\mu\|_{\infty} \sup _{t \in[-h, T]} \chi(\Omega(t)) \frac{1}{\eta}\left(1-E_{q}\left(-\eta t^{q}\right)\right)=\frac{\|\mu\|_{\infty}}{\eta} \sup _{t \in[-h, T]} \chi(\Omega(t)) .
\end{gathered}
$$

From the last estimate, we get

$$
\sup _{t \in[0, T]} \chi(G(\Omega)(t)) \leq \frac{\|\mu\|_{\infty}}{\eta} \sup _{t \in[-h, T]} \chi(\Omega(t)) .
$$


At the same time, notice that, from the definition of the multioperator $G$, it follows that

$$
\sup _{s \in[-h, 0]} \chi(G(\Omega)(s))=\sup _{t \in[T-h, T]} \chi(G(\Omega)(t)) \leq \sup _{t \in[0, T]} \chi(G(\Omega)(t)) .
$$

Taking into account estimates (21) and (22), we get

$$
\sup _{t \in[-h, T]} \chi(G(\Omega)(t)) \leq \frac{\|\mu\|_{\infty}}{\eta} \sup _{t \in[-h, T]} \chi(\Omega(t)),
$$

or that is the same

$$
\varphi(G(\Omega)) \leq \frac{\|\mu\|_{\infty}}{\eta} \varphi(\Omega)
$$

Conditions (29) and (20) obviously imply

$$
\varphi(\Omega)=0 .
$$

In the paper [5], it was shown that, on the interval [0,T]:

$$
\bmod _{C}\left(S \circ \mathcal{P}_{F}^{\infty}(\Omega)\right)=0 ;
$$

therefore, by representation (16), we also have

$$
\bmod _{C}(\widetilde{G}(\Omega))=0
$$

From the definition of $G$, it follows that

$$
\bmod _{C}(G(\Omega))=0
$$

implying, by (20),

$$
\bmod _{C}(\Omega)=0 .
$$

This means, by the Arzela-Ascoli theorem, that $\Omega$ is a relatively compact set, concluding the proof.

Now, we are in position to prove the main result of this paper.

Theorem 4. Under conditions $(A),(A 1),(F 1)-(F 4)$, if

$$
\frac{k}{\eta}<1
$$

where $k=\max \left\{\|\gamma\|_{\infty},\|\mu\|_{\infty}\right\}$, functions $\gamma$ and $\mu$ are from conditions (F3) and (F4) respectively, $\eta$ is the constant from condition (A1), then problems (7) and (8) have a solution.

Proof. Take arbitrary $x \in C([-h, T] ; E)$ and $y \in G(x)$, then, for some $\phi \in \mathcal{P}_{F}^{\infty}(x)$, we will have, for $t \in[0, T]$ the following estimate:

$$
\begin{gathered}
\|y(t)\|_{E} \leq \\
\leq\left\|\mathcal{G}(t)(I-\mathcal{G}(T))^{-1} \int_{0}^{T}(T-s)^{q-1} \mathcal{T}(T-s) \phi(s) d s+\int_{0}^{t}(t-s)^{q-1} \mathcal{T}(t-s) \phi(s) d s\right\|_{E} \leq \\
\|\mathcal{G}(t)\|\left\|(I-\mathcal{G}(T))^{-1}\right\| \int_{0}^{T}(T-s)^{q-1} \mathcal{T}(T-s)\left(\alpha(s)+\gamma(s)\left\|x_{t}\right\|_{\mathcal{C}}\right) d s+
\end{gathered}
$$




$$
\begin{gathered}
+\int_{0}^{t}(t-s)^{q-1}\|\mathcal{T}(t-s)\|\left(\alpha(s)+\gamma(s)\left\|x_{t}\right\|_{\mathcal{C}}\right) d s \leq \\
\leq \frac{E_{q}\left(-\eta t^{q}\right)}{1-E_{q}\left(-\eta T^{q}\right)} \int_{0}^{T}(T-s)^{q-1} E_{q, q}\left(-\eta(T-s)^{q}\right)\left(\alpha(s)+\gamma(s)\|x\|_{C([-h, T] ; E)}\right) d s+ \\
\int_{0}^{t}(t-s)^{q-1} E_{q, q}\left(-\eta(t-s)^{q}\right)\left(\alpha(s)+\gamma(s)\|x\|_{C([-h, T] ; E)}\right) d s \leq \\
\leq \frac{E_{q}\left(-\eta t^{q}\right)}{1-E_{q}\left(-\eta T^{q}\right)}\left(\|\alpha\|_{\infty}+\|\gamma\|_{\infty}\|x\|_{C([-h, T] ; E)}\right) \frac{1}{\eta}\left(1-E_{q}\left(-\eta T^{q}\right)\right) \\
+\left(\|\alpha\|_{\infty}+\|\gamma\|_{\infty}\|x\|_{C([-h, T] ; E)}\right) \frac{1}{\eta}\left(1-E_{q}\left(-\eta t^{q}\right)\right)= \\
=\left(\|\alpha\|_{\infty}+\|\gamma\|_{\infty}\|x\|_{C([-h, T] ; E)}\right) \frac{1}{\eta} .
\end{gathered}
$$

Notice that, by definition of $G$, the last estimate is also valid for $t \in[-h, 0]$.

Now, if we will take

$$
R \geq \frac{\|\alpha\|_{\infty} \eta^{-1}}{1-\|\gamma\|_{\infty} \eta^{-1}}
$$

then the inequality $\|x\|_{C([-h, T] ; E)} \leq R$ implies $\|G(x)\|_{C([-h, T] ; E)} \leq R$. Therefore, the multioperator $G$ transforms the closed ball $B_{R}(0) \subset C([-h, T] ; E)$ into itself. By Theorem $1, G$ has a fixed point, which is, by virtue of Theorem 2, a solution of problems (7) and (8).

\section{Example: A Periodic Problem for a Time-Fractional Diffusion System}

At the present time, the research of many authors (see [3] and the references therein) are devoted to the study of equations of the form

$$
D_{t}^{\alpha} y(x, t)=\frac{d^{2} y(x, t)}{d x^{2}}
$$

Since the order $\alpha$ of the derivative with respect to time in Equation (24) can be of arbitrary real order, including $\alpha=1$, it is called the fractional diffusion-wave equation. This name has been suggested by Mainardi (see [21]). For $\alpha=1$, Equation (24) becomes the classical diffusion equation; for, $0<\alpha<1$, we have so-called ultraslow diffiision. It is important that the fractional diffusion equation has been related to a dynamical process in fractal media: the order of the resulted equation depends on the fractal, which serves as a model of a porous material (see, e.g., [22]).

In our example, we consider such a fractional diffusion process subject to control effects.

Let $\mathbb{R}^{2}$ be the two-dimensional plane of points $\zeta=\left(\zeta_{1}, \zeta_{2}\right)$. As earlier, by $\mathbb{R}_{+}^{2}$, we denote the first quadrant of the plane defined by Formula (17). Denote by $L^{2}\left(\mathbb{R}_{+}^{2}\right)$ the Hilbert spaces of functions square summable on $\mathbb{R}_{+}^{2}$.

We will consider a time-fractional control system whose state will be described by the function $v:[-h, T] \times \mathbb{R}_{+}^{2} \rightarrow \mathbb{R}, v(t, \cdot) \in L^{2}\left(\mathbb{R}_{+}^{2}\right), t \in[-h, T]$. Similarly to the foregoing, let us denote by $\mathcal{C}:=C\left([-h, 0] ; L^{2}\left(\mathbb{R}_{+}^{2}\right)\right)$.

The control is characterized by $k$ sources of external influence whose properties at the moment $t \in[0, T]$ are dependent on the prehistory of the system. Their densities are described by the functions $\phi_{i}: \mathbb{R}_{+}^{2} \times \mathcal{C} \rightarrow \mathbb{R}, i=1, \ldots, k$ and the intensities of sources can be regulated by the controls $v_{i}:[0, T] \rightarrow \mathbb{R}$, $i=1, \ldots, k$, measurable functions satisfying the feedback condition with delay of the form

$$
\left.v(t)=\left(v_{1}(t), \ldots, v_{k}(t)\right) \in W\left(v_{t}\right)\right), \quad t \in[0, T],
$$


where $v_{t} \in \mathcal{C}$ is defined as the function $s \rightarrow v(t+s, \cdot), s \in[-h, 0]$ and $W$ is a u.s.c. multimap from $\mathcal{C}$ to Euclidean space $\mathbb{R}^{m}$ with convex closed values which is globally bounded:

$$
\|W(\xi)\| \leq \mathcal{W}
$$

for all $\xi \in \mathcal{C}$, where $\mathcal{W}>0$.

We will consider a time-fractional diffusion type feedback control system with delay governed by the following equation of the order $0<q<1$ :

$$
\begin{array}{ll}
\left.{ }^{C} D_{t}^{q} v(t, \zeta)=(\triangle-\eta) v(t, \zeta)+\sum_{i=1}^{k} v_{i}(t) \phi_{i}\left(\zeta, v_{t}\right)\right), & (t, \zeta) \in[0, T] \times \mathbb{R}_{+}^{2} \\
\frac{\partial}{\partial n} v(t, \zeta)=0, & (t, \zeta) \in[0, T] \times \partial \mathbb{R}_{+}^{2},
\end{array}
$$

where $\triangle=\frac{\partial^{2}}{\partial \zeta_{1}^{2}}+\frac{\partial^{2}}{\partial \zeta_{2}^{2}}$ is the Laplace operator, $\eta>0$.

For the above system, we will study the existence of a solution $v(t, \zeta)$ satisfying the periodic boundary value condition of the form

$$
v_{0}=v_{T} .
$$

Consider the differential operator of the form $A=\triangle-\eta I$ with the domain $H_{0}^{2}\left(\mathbb{R}_{+}^{2}\right)$, where $H_{0}^{2}$ denotes the Sobolev space of functions whose normal derivatives $\frac{\partial}{\partial n}$ are vanishing on the boundary. Then, as it is shown in [23], the boundary value problem

$$
\begin{gathered}
(\lambda I-A) u=f, \quad \lambda \geq 0, \\
\left.\frac{\partial}{\partial n} u\right|_{\Gamma}=0
\end{gathered}
$$

for $f \in L^{2}\left(\mathbb{R}_{+}^{2}\right), \Gamma=\partial \mathbb{R}_{+}^{2}$ is solvable and the operator associating to the function $f$ the solution $u$ is bounded. Moreover, since the set of functions with a finite support vanishing on a neighborhood of the boundary $\Gamma$ is densely embedded in $L^{2}\left(\mathbb{R}_{+}^{2}\right)$, by applying the Green formula to the expression $\int_{\Omega} u \triangle u d x$, where $\Omega=\mathbb{R}_{+}^{2}$, we get, for $\lambda>0$, the following estimate:

$$
(\lambda+\eta)\|u\|^{2} \leq(\lambda+\eta)\langle u, u\rangle-\int_{\Omega} u \triangle u d x=\langle(\lambda I-A) u, u\rangle \leq\|f\|\|u\|,
$$

where the norm and the scalar product are taken in $L^{2}\left(\mathbb{R}_{+}^{2}\right)$. Hence, for the resolvent $R(\lambda, A)=$ $(\lambda I-A)^{-1}$, the following estimate is true:

$$
\|R(\lambda, A)\| \leq \frac{1}{\lambda+\eta} \text { for } \lambda>0 .
$$

This means (see, e.g., [24] Corollary II.3.5) that the operator $A$ generates a strongly continuous semigroup $e^{A t}$ satisfying the estimate

$$
\left\|e^{A t}\right\| \leq e^{-\eta t}, \quad t \geq 0
$$

Notice that, since the resolvent $R(\lambda, A)$ is noncompact, the semigroup $e^{A t}$ is also noncompact.

Furthermore, we assume that functions $\phi_{i}, i=1, \ldots, k$ satisfy the following conditions:

$(\phi 1) \phi_{i}(\cdot, \xi): \mathbb{R}_{+}^{2} \rightarrow \mathbb{R}$ is measurable for all $\xi \in \mathcal{C} ;$

$(\phi 2)\left|\phi_{i}(\zeta, \xi)\right| \leq \omega_{i}(\zeta)$ for a.e. $\zeta \in \mathbb{R}_{+}^{2}$ and all $\xi \in \mathcal{C}$, where $\omega_{i} \in L_{+}^{2}\left(\mathbb{R}_{+}^{2}\right)$;

$(\phi 3)\left|\phi_{i}\left(\zeta, \xi_{1}\right)-\phi_{i}\left(\zeta, \xi_{2}\right)\right| \leq \mu_{i}\left\|\xi_{1}-\xi_{2}\right\|_{\mathcal{C}}$ for all $\xi_{1}, \xi_{2} \in \mathcal{C}$. 
Then, it is easy to see that the map $h: \mathcal{C} \times \mathcal{B}_{\mathcal{W}}\left(\mathbb{R}^{k}\right) \rightarrow L^{2}\left(\mathbb{R}_{+}^{2}\right)$, where $\mathcal{B}_{\mathcal{W}}\left(\mathbb{R}^{k}\right)=\left\{v \in \mathbb{R}^{k}:\|v\| \leq\right.$ $\mathcal{W}$, defined as

$$
h(\xi, v)(\zeta)=\sum_{i=1}^{k} v_{i} \phi_{i}(\zeta, \xi)
$$

is $\mu$-Lipschitz in $\xi$ with

$$
\mu=\mathcal{W} \sqrt{\sum_{i=1}^{k} \mu_{i}^{2}}
$$

and compact in $v$, i.e., the set $h\left(\xi, \mathcal{B}_{\mathcal{W}}\left(\mathbb{R}^{k}\right)\right)$ is relatively compact in $L^{2}\left(\mathbb{R}_{+}^{2}\right)$ for each $\xi \in \mathcal{C}$.

However, then, one can verify (see [18] Proposition 2.2.2) that the multimap $F: \mathcal{C} \rightarrow K v\left(L^{2}\left(\mathbb{R}_{+}^{2}\right)\right.$ )

$$
F(\xi)=h(\xi, W(\xi))
$$

satisfies conditions $(F 2)-(F 4)$ of Section $3\left(\right.$ with $\alpha(t) \equiv \omega:=\mathcal{W} \sqrt{\sum_{i=1}^{k}\left\|\omega_{i}\right\|_{L^{2}}^{2}}, \gamma(t) \equiv 0$ in condition (F3) and $\mu(t) \equiv \mu$ in condition (F4)).

Now, we can rewrite our system in the form of the following fractional-order functional differential inclusion in the Hilbert space $E=L^{2}\left(\mathbb{R}_{+}^{2}\right)$ :

$$
{ }^{C} D^{q} x(t) \in A x(t)+F\left(x_{t}\right), \quad t \in[0, T] .
$$

To verify condition (13), let us estimate the norm of the operator $\mathcal{G}(T)$. By using estimate (29), we have

$$
\|\mathcal{G}(T)\| \leq \int_{0}^{\infty} \xi_{q}(\theta)\left\|e^{A\left(T^{q} \theta\right)}\right\| d \theta \leq \int_{0}^{\infty} \xi_{q}(\theta) e^{-\eta T^{q} \theta} d \theta=E_{q}\left(-\eta T^{q}\right)<1 .
$$

Now, in accordance with Theorem 4, we conclude that, under condition

$$
\frac{\mu}{\eta}<1
$$

system (26) has a trajectory $v^{*}(t, \zeta)$ satisfying (28). The corresponding control may be taken as a measurable selection of the multifunction $W\left(v_{t}^{*}\right)$.

Author Contributions: Investigation, M.K., V.O., G.P. and J.-C.Y. These authors contributed equally to this work.

Funding: The work is supported by the joint Taiwan MOST-Russia RFBR grant 17-51-52022. The work of the first, second, and the third authors is supported by the Ministry of Education and Science of the Russian Federation in the frameworks of the project part of the state work quota (Project No 1.3464.2017/4.6). The work of the third author is supported by the RFBR Grant 19-31-60011.

Acknowledgments: The work on the paper was carried out during Valeri Obukhovskii's and Mikhail Kamenskii's visit to the Center for Fundamental Science, Kaohsiung Medical University and the Department of Applied Mathematics, National Sun Yat-Sen University, Kaohsiung, Taiwan in 2019. They would like to express their gratitude to the members of the Center and the Department for their kind hospitality.

Conflicts of Interest: The authors declare no conflict of interest.

\section{References}

1. Kilbas, A.A.; Srivastava, H.M.; Trujillo, J.J. North-Holland Mathematics Studies. In Theory and Applications of Fractional Differential Equations; Elsevier Science B.V.: Amsterdam, The Netherlands, 2006; Volume 204.

2. An, N.T.; Nam, N.M.; Qin, X. Solving k-center problems involving sets based on optimization techniques. J. Glob. Optim. 2019. [CrossRef]

3. Podlubny, I. Fractional Differential Equations; Academic Press: San Diego, CA, USA, 1999.

4. Zhou, Y. Fractional Evolution Equations and Inclusions: Analysis and Control; Elsevier Academic Press: London, UK, 2016. 
5. Kamenskii, M.; Obukhovskii, V.; Petrosyan, G.; Yao, J.C. On semilinear fractional order differential inclusions in Banach spaces. Fixed Point Theory 2017, 18, 269-292. [CrossRef]

6. Kamenskii, M.; Obukhovskii, V.; Petrosyan, G. Yao, J.C. Boundary value problems for semilinear differential inclusions of fractional order in a Banach space. Appl. Anal. 2018, 97, 571-591. [CrossRef]

7. Kamenskii, M.; Obukhovskii, V.; Petrosyan, G.; Yao, J.C. On approximate solutions for a class of semilinear fractional-order differential equations in Banach spaces. Fixed Point Theory Appl. 2017, 2017, 28. [CrossRef]

8. Ke, T.D.; Loi, N.V.; Obukhovskii, V. Decay solutions for a class of fractional differential variational inequalities. Fract. Calc. Appl. Anal. 2015, 18, 531-553.

9. Ke, T.D.; Obukhovskii, V.; Wong, N.C.; Yao, J.C. On a class of fractional order differential inclusions with infinite delays. Appl. Anal. 2013, 92, 115-137. [CrossRef]

10. Abbas, S.; Benchohra, M.; Hamidi, N. Stability for impulsive fractional differential inclusions via Picard operators in Banach spaces. J. Nonlinear Funct. Anal. 2018, 2018, 44.

11. Ahmad, B.; Alsaedi, A.; Ntouyas, S.K. Multi-term fractional boundary value problems with four-point boundary conditions. J. Nonlinear Funct. Anal. 2019, 2019, 40.

12. Obukhovskii, V.; Yao, J.C. Some existence results for fractional functional differential equations. Fixed Point Theory 2010, 11, 85-96.

13. Benedetti, I.; Obukhovskii, V.; Taddei, V. On generalized boundary value problems for a class of fractional differential inclusions. Fract. Calc. Appl. Anal. 2017, 20, 1424-1446. [CrossRef]

14. Zhang, Z.; Liu, B. Existence of mild solutions for fractional evolution equations. Fixed Point Theory 2014, 15, 325-334.

15. Zhou, Y.; Jiao F. Existence of mild solutions for fractional neutral evolution equations. Comput. Math. Appl. 2010, 59, 1063-1077. [CrossRef]

16. Wang, R.N.; Chen, D.H.; Xiao, T.J. Abstract fractional Cauchy problems with almost sectorial operators. J. Differ. Equ. 2012, 252, 202-235. [CrossRef]

17. Gorenflo, R.; Kilbas, A.A.; Mainardi, F.; Rogosin, S.V. Mittag-Leffler Functions, Related Topics and Applications; Springer: Berlin/Heidelberg, Germany, 2014.

18. Kamenskii, M.; Obukhovskii, V.; Zecca, P. de Gruyter Series in Nonlinear Analysis and Applications. In Condensing Multivalued Maps and Semilinear Differential Inclusions in Banach Spaces; Walter de Gruyter: Berlin, Germany; New York, NY, USA, 2001; Volume 7.

19. Górniewicz, L. Topological Fixed Point Theory and Its Applications. In Topological Fixed Point Theory of Multivalued Mappings, 2nd ed.; Springer: Dordrecht, The Netherlands, 2006; Volume 4.

20. Borisovich, Y.G.; Gelman, B.D.; Myshkis, A.D.; Obukhovskii ,V.V. Introduction to the Theory of Multivalued Maps and Differential Inclusions, 2nd ed.; Librokom: Moscow, Russia, 2011. (In Russian)

21. Mainardi, F. On the initial value problem for the fractional diffusion-wave equation. In Waves and Stability in Continuous Media; Rionero, S., Ruggeri, T., Eds.; World Scientific: Singapore, 1994; pp. 246-251.

22. Nigmatullin, R.R. The realization of the generalized transfer equation in a medium with fractal geometry. Phys. Status Solidi B 1986, 133, 425-430. [CrossRef]

23. Titchmarsh, E.C. Eigenfunction Expansions Associated with Second-Order Differential Equation; Clarendon Press: Oxford, UK, 1958; Volume 2.

24. Engel, K.-J.; Nagel, R. A Short Course on Operator Semigroups; Springer: New York, NY, USA, 2006.

(C) 2019 by the authors. Licensee MDPI, Basel, Switzerland. This article is an open access article distributed under the terms and conditions of the Creative Commons Attribution (CC BY) license (http:/ / creativecommons.org/licenses/by/4.0/). 